\title{
Bienestar del cuidador/a familiar del adulto mayor con dependencia funcional: una perspectiva de género
}

Welfare of the functional dependence elderly family caregiver:

a gender perspective

\section{Bem estar dos cuidadores de idosos com deficiência física: nas perspectivas do homem e da mullher}

Aurora Félix Alemán ${ }^{1}$; Rosa María Aguilar Hernández²; Ma. Luz Martínez Aguilar³; Hermelinda Avila Alpirez; Laura Vázquez Galindo ${ }^{4}$; Gustavo Gutiérrez Sánchez ${ }^{4}$

${ }^{1}$ CD en Enfermería y Cultura de los Cuidados. ${ }^{2}$ Dra. en Educación Internacional.

${ }^{3}$ Dra. en Enfermería y Cultura de los Cuidados. ${ }^{4}$ Dr. en Psicología

Cómo citar este artículo en edición digital: Félix Alemán A; Aguilar Hernández RMa; Martínez Aguilar MáL; Avila Alpírez H; Vázquez Galindo L; Gutiérrez Sánchez G. (2012) Bienestar del cuidador/a familiar del adulto mayor con dependencia funcional: una perspectiva de género. Cultura de los Cuidados. (Edición digital) 16, 33. Disponible en: <http://dx.doi.org/10.7184/ cuid.2012.33.011>

Correspondencia: Aurora Feliz Alemán. Playa Miramar No. 22 e/Playa Mocambo y Priv. Ciro R. de la Garza. C. P. 87470. Matamoros, Tamaulipas México.E-mail: afelix@uat.edu.mx. Tel: (868) 812-27-16

Recibido: 011/11/2011/ Aceptado: 10/03/2012

*Este trabajo se deriva del proyecto Bienestar del cuidador familiar del adulto mayor: una perspectiva de género, con financiamaiento del Programa Integral de Fortalecimiento Institucional/2008 otorgado al Cuerpo Académico salud y Comportamiento Humano de la Unidad Académica Multidisciplinaria Matamoros-UAT.

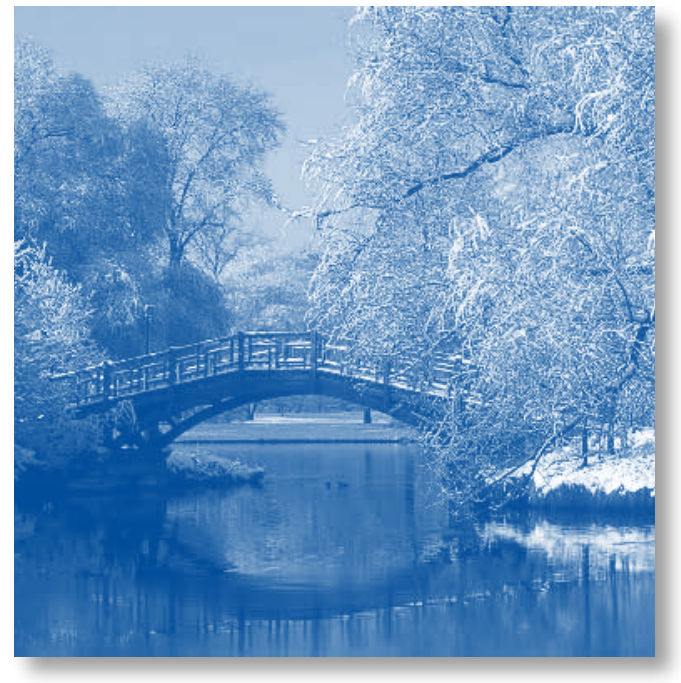

ABSTRACT

Family carers of the elderly dependent (CFAMD), vulnerable group susceptible to damage health condition; is aggravated by gender, as reflected in dimensions of welfare. Objective: To identify the welfare of the func- tional dependence elderly family caregiver with a gender perspective. Method: A descriptive, analytical and correlational study. Sample: 300 CFAMDF, Matamoros Tamaulipas, Mexico. Questionnaires: Sociodemographic and care data, Barthel Index and Quality of life, the latter with Cronbach alpha of 0.69. Chi-square and Pearson correlation coefficient tests. Results: Description of CFAMDF, women $(85.0 \%)$, average age 42 , married/ cohabiting $(65.0 \%)$, son/daughter $(61.4 \%)$, household work (66.3\%), unemployed (49.3\%) or income limited (50.7\%). Women with more time and dedication to care as well as greater involvement in almost all dimensions of wellness. CFAMDF features: kinship (daughters) and occupation (household work) associated with dimensions of wellness. Welfare unaffected when only caring the elderly. Conclusion: CFAMDF was characterized as involvement in 
women with dimensions of welfare associated with older age, functional dependency and greater time caring the elderly. The CFAMDF women prevalence reflects the social conditioning of gender.

Keywords: Welfare. Caregiver. Elderly. Gender.

\section{RESUMO}

Cuidadores Familiares de Idosos com Deficiência Física (CFAMD), população vulnerável suscetível a danos de saúde; agravos nas diferenças de gênero de cuidados e reflexos no bem estar. Objetivo: mensurar o bem estar dos cuidadores de idosos com deficiência física, nas perspectivas do homem e da mulher. Método: estudo descritivo, analítico e correlacional. A amostra é de 300 usuários do serviço CFAMDF, na cidade de Matamoros Tamaulipas, no México. Instrumentos: dados sociodemográficos e cuidados, índice de Barthel e Qualidade Vida, cruzados com Alpha de Cronbach 0.69. Testes: Qui-Quadro e coeficiente de correlação de Person. Resultados: CFAMD, mulheres (85,0\%), em média 42 anos, casadas/união estável (65,0\%), com filho/a (61,4\%), donas de casa (66,3\%), desempregadas $(49,3 \%)$, nível socioeconômico baixo $(50,7 \%)$. Constatou-se que mulheres com mais tempo e dedicação para o cuidado, apresentaram maior impacto em quase todas as esferas de cuidados e bem estar. Características CFAMDF: parentesco (filhas) e ocupação (donas de casa) estão associadas às esferas de bem-estar. As que dedicam-se somente aos cuidados aos idosos tiveram apresentaram danos no bem-estar. Conclusão: CFAMDF se caracterizou por mulheres cuidadoras que, quanto maior a idade das pessoas com deficiência físicas cuidadas, bem como o maior tempo de cuidados em AM, maior sãos os danos nas dimensões de seu bem-estar. Contudo o predomino foi de mulheres CFAMDF, tendo como reflexos os fatores sociais constituídos.

Palavras-chave: Cuidador familiar; Idosos; Perspectiva de gênero.

\section{RESUMEN}

Cuidadores familiares adultos mayores dependientes (CFAMD), grupo vulnerable, susceptible de daños a salud; se agrava por condición de género, reflejado en dimensiones del bienestar. Objetivo: identificar bienestar del cuidador/a familiar del adulto mayor con dependencia funcional, con perspectiva de género. Método: estudio descriptivo, analítico y correlacional. Muestra 300 CFAMDF, Matamoros Tamaulipas, México. Cuestionarios: Datos Sociodemográficos y del Cuidado, Índice de Barthel y Calidad Vida, éste con alpha de Cronbach 0.69. Pruebas chi-cuadrada y coeficiente de correlación de Pearson. Resultados: CFAMDF, mujeres (85.0\%), media 42 años, casadas/unión libre (65.0\%), hijo/a (61.4\%), labores hogar (66.3\%), sin trabajo (49.3\%) o ingresos limitados (50.7\%). Mujeres con más tiempo y dedicación al cuidado, mayor afectación en casi todas las dimensiones del bienestar. Características CFAMDF: parentesco (hijas) y ocupación (labores del hogar) asociadas con dimensiones del bienestar. Dedicación sólo al cuidado AM sin afectación del bienestar. Conclusión: CFAMDF se caracterizó como mujeres con afectación en dimensiones del bienestar asociado con mayor edad, dependencia funcional y mayor tiempo al cuidado AM. Predominio mujeres CFAMDF refleja el condicionamiento social de género.

Palabras clave: Bienestar. Cuidador. Adulto mayor. Género. 


\section{INTRODUCCIÓN}

La atención a la salud como derecho universal debe ofrecerse sin distinción, en particular a grupos vulnerables como lo es el de Cuidador/a Familiar del Adulto Mayor con Dependencia Funcional (CFAMDF).

El grupo Adulto Mayor (AM) se encuentra en rápida expansión y amerita atención (Olshansky, Biggs, Achenbaum, Davison, Fried, Gutman, Kalache, Khaw, Fernandez, Rattan, Guimarães, Milner y Butler, 2011) y cuidados por su familia, que se agudizan por el deterioro progresivo y daños a la salud, lo que da lugar a la dependencia funcional (Aguilar, Félix, Martínez, Vega, Quevedo y Gutiérrez, 2007, 2009; González y Ham-Chande, 2007). Las exigencias del cuidado aumentan con el nivel de dependencia del AM lo cual somete a riesgo de daño a la salud al CFAMDF (Ministerio de Trabajo y Asuntos Sociales, 2005; Montes de Oca y Hebrero 2006).

El Cuidador/a Familiar del Adulto Mayor con Dependencia Funcional es la persona, en relación de parentesco, hombre o mujer, mayor de 18 años, que atiende, apoya y supervisa sus necesidades básicas de la vida diaria, toma decisiones y asume su cuidado de salud integral. El CFAMDF se caracteriza como: mujer, principalmente hija, en edad media de la vida con proximidad o dentro del grupo AM, casada o en unión libre, dedicada al hogar, con baja escolaridad y percepción económica (Ministerio de Trabajo y Asuntos Sociales, 2005; Nigenda, López-Ortega, Matarazzo y Juárez-Ramírez, 2007; Rojas, 2007; Espinosa, Mendez, Lara y Rivera, 2009; Luengo, Araneda y López, 2010; Yonte, Urién, Martín y Montero, 2010).

La salud es un estado de completo bienestar físico, mental y social, y no solamente la ausencia de enfermedades (OMS, 2006); y en virtud de que, en México, la Constitución Po- lítica establece que toda persona tiene derecho a un medio ambiente adecuado para su desarrollo y bienestar (Instituto de Investigaciones Jurídicas, 2011), los profesionales del cuidado deben contribuir a su cumplimiento, mediante la identificación, estudio y atención a los grupos en riesgo. El bienestar de los CFAMDF se definió para este estudio como la adaptación e integración de las dimensiones física, psicológica, social y espiritual en el contínuum saludenfermedad.

El género es una construcción simbólica conformada de atributos asignados a las personas a partir del sexo como las características biológicas, físicas, económicas, sociales, psicológicas, eróticas, jurídicas, políticas y culturales (Lagarde, 2001). La equidad de género es una condición necesaria para el goce de la salud como derecho constitucional. De acuerdo con la teoría de género el sistema de organización patriarcal que impera en el contexto mexicano asigna por género las funciones productivas y reproductivas (Cazés, 2000). Las reproductivas son asignadas socialmente a la mujer, como un deber ser y vividas como condicionamiento social y moral; entre las que se encuentran el cuidado del hogar y los dependientes en la familia: niños, enfermos y adultos mayores. Por estas actividades la mujer no espera remuneración económica ni reconocimiento social ya que las mujeres son en función de otros. Ellas destinan sus energías vitales en la satisfacción de las necesidades de los otros de quienes se complementan (Lagarde, 2001, 2005). Las mujeres cuidadoras se entregan al cuidado con agrado por la satisfacción del deber cumplido lo cual no sucede en el cuidado del AM por el progresivo deterioro de su salud.

El problema de género es mundial, con mayor apego en culturas latinas, en donde las mujeres cumplen con el deber moral de cuidar 
a sus padres (Robles, Vázquez, Reyes y Orozco, 2007), con abnegación buscan la satisfacción de las necesidades de ellos en perjuicio de las propias (Díaz-Guerrero, 2003; Lagarde, 2005; Maqueira, 2006). Esta asignación tradicional les ha hecho dueñas, expertas y sucesoras del cuidado (Heierle, 2004; Bover, 2006). Por ello, el AM, llegado el momento, espera y/o pide ser cuidado por su hija, esposa u otra mujer en el hogar o donde sea necesario (Instituto de Mayores y Servicios Sociales, 2005; Robles y cols., 2007).

El cuidado sostenido del AM en el hogar requiere cada vez de más tiempo, conocimiento, fortaleza física y emocional, lo que supera las expectativas de las mujeres. Ellas asumen el cuidado: sin preparación, adquieren habilidad en condición de estrés (Schumacher, Beck y Marren, 2006; Barrera, Pinto y Sánchez, 2006; Roberto y Jarrott, 2008), sin apoyo social para el cuidado por lo que se considera un trabajo invisible (Nigenda y cols., 2007; Congreso General de los Estados Unidos Mexicanos, 2009), ocupadas en una segunda o tercera jornada, sin remuneración por el cuidado (Robles, 2003; Instituto Nacional de Estadística Geografía e informática, 2007), sin participación de la pareja en el cuidado del hogar e hijos, sin apoyo de su familia extendida (Nigenda y cols, 2007), con alta implicación en la responsabilidad a expensas de su propio bienestar y postergando sus proyectos, anhelos y motivaciones cotidianas (Mier, Romeo, Canto y Mier, 2007; Roberto y Jarrott, 2008; Uribe, 1999).

En este contexto, además de su caracterización como grupo, el de CFAMDF no sólo es susceptible de daño a la salud y ser sujeto de atención, sino de convertirse en futuro sujeto del cuidado; por lo que debería ser considerado como grupo vulnerable en los programas sociales, de salud y de investigación. Asimismo los profesionales de la salud, particularmente enfermería requieren la formación acorde a esa situación que les permita atender con efectividad a CFAMDF como sujeto de atención y sujeto del cuidado que resulte en su bienestar y el de su familiar adulto mayor (Salas, 2006).

El bienestar del CFAMDF en su condición de género y en las dimensiones del bienestar: física, psicológica, social y espiritual, no ha sido estudiado en México, por lo que se realizó un estudio a partir de la pregunta ¿Cómo influye el género en el bienestar del cuidador/a familiar del adulto mayor con dependencia funcional en Matamoros, Tamaulipas México? Con el objetivo de identificar el bienestar del cuidador/a familiar del adulto mayor con dependencia funcional, con una perspectiva de género. Este conocimiento permitirá planear intervenciones de enfermería que impacten en las dimensiones del bienestar en su condición de CFAMDF.

\section{MATERIAL Y MÉTODO}

Estudio descriptivo, analítico y correlacional. Muestra por conveniencia de 300 cuidadores/as principales de un adulto mayor con dependencia funcional, residentes en diferentes sectores del área urbana de Matamoros Tamaulipas, México, mayores de 18 años, con más de seis meses de manera continua en el cuidado y sin pago.

Instrumentos: Cuestionario Datos Sociodemográficos y del Cuidado, elaborado ex profeso; Índice de Barthel, para medir la dependencia funcional del adulto mayor, de Lowen y Anderson, referidos por Cid-Ruzafa y Damián-Moreno (1997) quienes encontraron alpha de Cronbach de 0.86 -0.92 y Calidad Vida para medir el bienestar y sus dimensiones en el cuidador/a familiar de Ferrell y Grant (s.f.) con alpha de Cronbach 0.69. Es un instrumento 


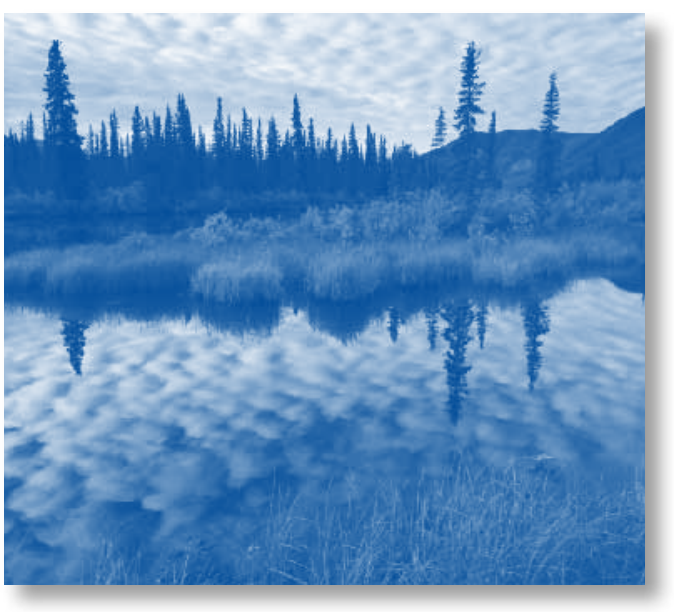

ordinal, escala tipo Likert de 37 reactivos que mide la calidad de vida a través de la exploración del bienestar en las dimensiones física, psicológica, social y espiritual. La escala es del cero al 10. Cero es el peor resultado y diez es el mejor, sin embargo en algunos reactivos esto es a la inversa: 1-4, 6, 13-20, 22, 24-29 y 33 . Procedimientos: se elaboró instructivo de cada instrumento para la capacitación y conducción de encuestadores: prestatario de servicio social y alumnos de la carrera de enfermería de la Unidad Académica Multidisciplinaria Matamoros, de la Universidad Autónoma de Tamaulipas (UAMM-UAT). La retroalimentación de la prueba piloto permitió uniformar la comprensión de las preguntas. Mediante visita domiciliaria se eligieron a cuidadores/ as con los criterios establecidos, previa firma del consentimiento informado para su participación. Para el procesamiento y análisis de los datos se utilizó el paquete estadístico SPSS, versión 15 , se aplicaron las pruebas estadísticas: chi-cuadrada y coeficiente de correlación de Pearson. El estudio se realizó apegado a lo dispuesto en el Reglamento de la Ley General de Salud en Materia de Investigación para la Salud (Secretaría de Salud, 1988) y con la aprobación del Comité de Investigación y Ética de la UAMM-UAT.

\section{RESULTADOS}

El grupo estudiado se caracterizó como: mujeres $(85.0 \%)$, en etapa media de la vida (media de 42años) o próximas a la etapa AM, casadas o en unión libre (65.0\%), hijo/a (61.4\%), baja escolaridad (media 7.7), labores del hogar (66.3\%), sin actividad productiva (49.3\%) o con ingresos limitados (50.7\%).

En la asociación de género con la situación laboral, horas laboral, horas al cuidado y tiempo al cuidado se encontró que las mujeres llevan más tiempo y dedican más horas al cuidado. La mitad de las mujeres no tenían actividad laboral, sin embargo de las que trabajaban ocho le dedicaron más tiempo que cualquier hombre. El cuidador/a familiar presentó un mejor bienestar en la medida que se dedicaba sólo al cuidado del AM.

El bienestar del CFAMDF presentó mayor afectación del bienestar en la dimensión espiritual en casi todas las características sociodemográficas y del cuidado, seguido de la dimensión física y psicológica; la social es la menos afectada. Las características del CFAMDF que más asociación mostraron con las dimensiones del bienestar fueron parentesco y ocupación (Tabla 1).

Se encontró un alto grado de asociación entre el bienestar y sus dimensiones. La afectación en las dimensiones del bienestar del CFAMDF mostró asociación con el nivel de dependencia del AM (coeficiente de correlación negativos). El Bienestar Espiritual es el indicador que menor asociación presentó con las demás dimensiones (Tabla 2).

\section{DISCUSIÓN}

Las características del CFAMDF, en las que predomina el género femenino, estuvieron asociadas con las dimensiones del bienestar lo cual refleja afectación en su condición de mu- 


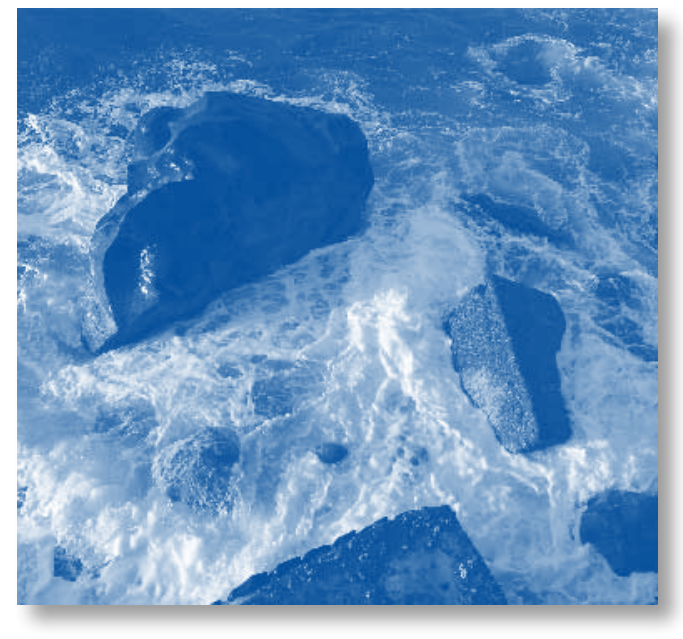

jer que se explican con perspectiva de género.

A las mujeres corresponde las funciones reproductivas que incluye el cuidado de la salud familiar (Lagarde, 2001), además del AM. En este estudio, el género se asoció con el bienestar en la dimensión espiritual. El complemento de las mujeres es el esposo y/o pareja con un sentido de propiedad (Lagarde, 2001), y condicionadas para su servicio lo que las somete en el dilema de cuidar a sus padres y de cumplir además como esposas. En este estudio predominó el estado civil casada, que se asoció con la dimensión espiritual.

Las mujeres son las encargadas de las funciones reproductivas (Lagarde, 2001). Lo esperado socialmente es que la mujer, por su condición de género se dedique al hogar. En este estudio la mayor ocupación fue el hogar, que se asoció con las dimensiones psicológicas, sociales y espirituales. Los hombres son los encargados de las funciones productiva (Lagarde, 2001), sin embargo la mitad de las mujeres de este estudio tienen, además, trabajo productivo, que aunado a sus múltiples roles incrementa su carga, esta característica se asoció con las dimensiones psicológica y espiritual, asimismo horas laboral con las dimensiones física y espiritual.
Las mujeres son en función de otros (Lagarde, 2001), sienten satisfacción del deber cumplido en beneficio de las personas a su cuidado, quienes son frecuentemente los padres y por su compromiso moral se ve mayormente implicada en el cuidado. En este estudio la relación de parentesco que predominó fue el de hija del AM la cual se asoció con las dimensiones física, psicológica y espiritual. La energía vital de las mujeres deben destinarse a satisfacer las necesidades vitales de los otros (Lagarde, 2001). Las mujeres cuidan con un sentido de responsabilidad, aunque en ello entregan la vida sobre todo cuando se trata del cuidado al AM con dependencia progresiva para el que se requiere mayor tiempo, dinero y esfuerzo. En este estudio las horas al cuidado se asoció con la dimensión psicológica; el ingreso económico con las dimensiones física y espiritual, la edad del AM con la dimensión física, y el nivel de dependencia del AM con las dimensiones física y social.

\section{CONCLUSIÓN}

Las características sociodemográficas del cuidador/a familiar y del cuidado del adulto mayor con dependencia funcional que se encontraron en el estudio, permitió identificar que las CFAMDF son un grupo en desventaja social y de salud en las que su ocupación, el parentesco y nivel de dependencia del AM afectan su bienestar mayormente en la dimensión espiritual y en menor medida la dimensión social.

\section{SUGERENCIA}

Incorporar la perspectiva de género en la formación profesional de enfermería para brindar un cuidado de calidad en este grupo vulnerable. 


\section{REFERENCIAS}

- Aguilar RM, Félix A, Martínez ML, Vega MC, Quevedo MM, Gutiérrez G. Dependencia funcional y género del adulto mayor. Desarrollo Científ Enferm. 2007 noviembre-diciembre, 15(10): 436-439.

- Aguilar RM, Félix A, Martínez ML, Vega MC, Quevedo MM, Gutiérrez G. Actividades de vida del adulto mayor en interacción con la familia. Desarrollo Científ Enferm. 2009, Julio 17(6).

- Barrera L, Pinto N, Sánchez B.Cuidando a los Cuidadores. Un programa de apoyo a familiares de personas con enfermedad crónica. Index de Enfermería 2006, XV (5253), 54-58.

- Bover A. El impacto de cuidar en el bienestar percibido por mujeres y varones de mediana edad: una perspectiva de género. Enfermería Clínica , 2006. 16 (2), 69-76.

- Cazés D. La perspectiva de género. Consejo Nacional de Población.(México). 2000.

- Cid- Ruzafa J, Moreno J. Valoración de la discapacidad física: El índice de Barthel. Rev Esp Salud Pública. 1997; 71(2): 127-137.

- Congreso General de los Estados Unidos Mexicanos. (24 de Junio de 2009). Ley de los Derechos de las Personas Adultas Mayores. Recuperado el 23 de Mayo de 2010, de sitio Web de la Cámara de Diputados del H. Congreso de la Unión: http://www.diputados.gob.mx/LeyesBiblio/ pdf/245.pdf

- Díaz R. La estructura psicológica de la familia mexicana. Descubrimiento de la etnopsicología. 6a ed. (México) Trillas. 2003.

- Espinosa E, Mendez V, Lara R, Rivera P. Factores asociados al nivel de sobrecarga de los cuidadores informales de adultos mayores dependientes, en control en el consultorio "Jose Durán Trujillo", San Carlos, Chile. Theoria. $2009 ; 18(1): 69-79$

- Ferrell R, Grant, M. (s.f).Quality of Life Family Version. National Medical Center and Beckman Research Institute. Consultado el 15 de mayo de 2007 de http://prc.coh. org/pdf/Quality of Life Family Version.pdf

- González CA, Ham R. Funcionalidad y salud: Una tipología del envejecimiento en México. Salud Pública Mex.
2007; 49 4: 448-458.

- Nigenda G, López M, Matarazzo C, Juárez C. La atención de los enfermos y discapacitados en el hogar. Retos para el sistema de salud mexicano. salud pública Méx . 2007 49, 4, julio-agosto de 286-294

- Heierle C. Cuidando entre cuidadores: intercambio de apoyo en la familia. Index , 2004. 159.

- Instituto de Investigaciones Jurídicas de la UNAM. Legislación Federal. Constitución Política de los Estados Unidos Mexicanos. Título $1^{\circ}$. Artículo 4. Vigente al 1 de septiembre de 2011. Consultado el 15 de septiembre de 2011. Disponible en: http://info4.juridicas.unam.mx/ ijure/fed/9/5.htm?s

- Instituto de Mayores y Servicios Sociales [IMSERSO]. (2005). Cuidados a las personas mayores en los hogares españoles. El entorno familiar. (M. d. sociales, Ed.) Recuperado el 26 de Febrero de 2010, de sitio Web del IMSERSO: http://www.inforesidencias.com/docs_pago/ imserso-cuidados-mayores.pdf

- Instituto Nacional de Estadística Geografía e informática. Mujeres y hombres en México. $11^{\circ}$. ed. (México). INEGI 2007.

- Lagarde M. Género y feminismo. Desarrollo humano y democracia. 3a . ed. (España):horas y HORAS. 2001.

- Lagarde M. Los cautiverios de las mujeres: madresposas, monjas, putas, presas y locas. $4^{\mathrm{a}}$. ed. (México): Universidad Nacional Autónoma de México (2005).

- Luengo C, Araneda E, López G. Factores del cuidador familiar que influyen en el cumplimiento de los cuidados básicos del usuario postrado. Index Enferm. 2010; 19(1): 14-18.

- Maquieira V. Mujeres, globalización y derechos humanos. España: (2006). Cátedra.

- Mier I, Romero Z, Canto A Mier R. Interpretando el cuidado. Por qué cuidan sólo las mujeres y qué podemos hacer para evitarlo. Revista de servicios sociales 2007 (42), 29-38.

- Ministerio de Trabajo y Asuntos Sociales. Atención a las personas en situación de dependencia en España. Libro Blanco. España: Secretaría de Estado y Servicios Sociales, Familias y Discapacidad; 2005.

- Montes V, HebreroM. Eventos cruciales y ciclos familia- 
res avanzados: el efecto del envejecimiento en los hogares de México. Papeles de Población 2006.(050), 97-116. - Olshansky J, Biggs S, Achenbaum A, Davison C, Fried L, Gutman G, Kalache A, Khaw T, Fernandez A, Rattan S, Guimarães M, Milner C, Butler N. Global Policy. The Global Agenda Council on the Ageing Society: Policy Principles. Political Science. 2011 2, 1, 97-105.

- Organización Mundial de la Salud. Documentos Básicos Suplemento de la 45a . Edición, octubre de 2006. Constitución de la Organización Mundial de la Salud. Consultado el 15 de noviembre de 2008. Disponible en: http:// www.who.int/governance/eb/who_constitution_sp.pdf

- Roberto A, Jarrott E. Family caregivers of older adults: A life span perspective. Family Relations 2008. 57 (1), 100-111.

- Robles L. Doble o triple jornada: el cuidado a enfermos crónicos. En Estudios de hombre 17. México. 2003.

- Robles L, Vázquez F, Reyes L,Orozco I. Miradas sobre la vejez. Un enfoque antropológico. Religión y sociedad, 2007. 19 (40), 195-204

- Rojas V. Asociación entre la habilidad de cuidado del cuidador, el tiempo de cuidado y el grado de dependencia del adulto mayor que vive situación de enfermedad crónica, en la ciudad de Girardot. av. enferm. 2007; 25(1): 33-45.

- Salas PM. La ciencia de los cuidados, marco investigativo para alcanzar el éxito de la calidad de vida en el envejecimiento. Cultura de los cuidados. 2006,. X(19): 73-78.

- Schumacher B, Marren M. Family caregivers: Caring for older adults, working with their families. American Journal of Nursing ,2006.106 (8), 40-49.

- Secretaría de Salud. Reglamento de la Ley General de Salud en materia de investigación para la salud (México). Secretaría de Salud 1988. Disponible en: <http://www. salud.gob.mx/unidades/cdi/nom/compi/rlgsmis.html> [Consultado el 18.01.2010].

- Uribe M.El auto cuidado y su papel en la promoción de las Salud. Investigación y Educación en Enfermería 1999 17 (2). 109-118.

- Yonte F,Urién Z, Martín M, Montero R. Sobrecarga del cuidador principal. Rev enfer C y L. 2010, 2(1): 59-69.

\section{TABLA 1}

Dimensiones del bienestar en función de las características sociodemográficas y del cuidado del cuidador/a familiar

Significativos con $\mathrm{p}<0.10$

Coeficiente de correlación de Pearson En negrilla niveles de significancia

\begin{tabular}{|lllll|}
\hline & \multicolumn{4}{c|}{ Dimensiones del bienestar } \\
\cline { 2 - 5 } Características del cuidador & Físico & Psicológico & Social & Espiritual \\
\hline Género & 0.2526 & 0.2119 & 0.3105 & $\mathbf{0 . 0 0 8 7}$ \\
Parentesco & $\mathbf{0 . 0 2 7 0}$ & $\mathbf{0 . 0 9 3 9}$ & 0.1195 & $\mathbf{0 . 0 0 1 5}$ \\
Estado civil & 0.7259 & 0.7806 & 0.7900 & $\mathbf{0 . 0 8 2 4}$ \\
Ocupación & 0.5366 & $\mathbf{0 . 0 3 9 3}$ & $\mathbf{0 . 0 1 7 5}$ & $\mathbf{0 . 0 1 8 1}$ \\
Horas laboral & $\mathbf{0 . 0 1 1 5}$ & 0.4181 & 0.1859 & $\mathbf{0 . 0 0 0 1}$ \\
Situación laboral & 0.9786 & $\mathbf{0 . 0 4 5 4}$ & 0.2023 & $\mathbf{0 . 0 1 2 0}$ \\
Ingreso económico & $\mathbf{0 . 0 5 7 0}$ & 0.9863 & 0.8700 & $\mathbf{0 . 0 2 9 3}$ \\
Horas al cuidado & 0.4852 & $\mathbf{0 . 0 3 9 0}$ & 0.7613 & 0.5026 \\
Edad del adulto mayor & $\mathbf{0 . 0 8 7 8}$ & 0.2248 & 0.4490 & 0.9292 \\
$\begin{array}{l}\text { Nivel de dependencia del adulto } \\
\text { mayor(Índice de Barthel) }\end{array}$ & $\mathbf{0 . 0 0 0 2}$ & 0.2439 & $\mathbf{0 . 0 0 0 8}$ & 0.2207 \\
\hline
\end{tabular}

\begin{tabular}{|lccccc|}
\hline Dimensiones del bienestar & Físico & Psicológico Social & Espiritual Bienestar \\
\hline Bienestar Psicológico & 0.587 & & & & \\
Bienestar Social & 0.575 & 0.733 & & & \\
Bienestar Espiritual & 0.353 & 0.448 & 0.438 & & \\
Bienestar & 0.734 & 0.921 & 0.878 & 0.635 & \\
$\begin{array}{l}\text { Nivel de dependencia del adulto } \\
\text { mayor (Índice de Barthel) }\end{array}$ & -0.208 & -0.113 & -0.209 & -0.078 & -0.179 \\
\hline
\end{tabular}

\section{TABLA 2}

Dimensiones del bienestar del cuidador/a familiar y nivel de dependencia del adulto mayor

${ }^{\star}$ Coeficientes mayores en valor absoluto a 0.113 son estadísticamente significativos con $\mathrm{p}=0.05$ Coeficiente de correlación de Pearson. 